\title{
Evidence-Based Study to Compare Daodi Traditional Chinese Medicinal Material and Non-Daodi Traditional Chinese Medicinal Material
}

\author{
Xingyue Yang $\mathbb{D},{ }^{1,2}$ Xin Tian, ${ }^{3}$ Yannan Zhou, ${ }^{3}$ Yali Liu $\mathbb{D},{ }^{4,5}$ Xinlong Li, ${ }^{4}$ Tingting Lu, ${ }^{5}$ \\ Changhe $\mathrm{Yu}^{6}$ and Liyun $\mathrm{He} \mathbb{1}^{4}$ \\ ${ }^{1}$ Beijing University of Chinese Medicine, Beijing 100700, China \\ ${ }^{2}$ State Key Laboratory Breeding Base of Dao-Di Herbs, China Academy of Chinese Medical Sciences, Beijing 100700, China \\ ${ }^{3}$ The First Clinical Medical College of Lanzhou University, Lanzhou 730000, China \\ ${ }^{4}$ Institute of Basic Research in Clinical Medicine, China Academy of Chinese Medical Sciences, Beijing 100700, China \\ ${ }^{5}$ Evidence-Based Medicine Center, School of Basic Medical Sciences, Lanzhou University, Lanzhou 730000, China \\ ${ }^{6}$ Department of Tuina and Pain Management, Dongzhimen Hospital, Beijing University of Chinese Medicine, \\ Beijing 100700, China \\ Correspondence should be addressed to Liyun He; hely3699@163.com
}

Received 23 August 2017; Accepted 19 November 2017; Published 22 January 2018

Academic Editor: Rentian Wu

Copyright (c) 2018 Xingyue Yang et al. This is an open access article distributed under the Creative Commons Attribution License, which permits unrestricted use, distribution, and reproduction in any medium, provided the original work is properly cited.

\begin{abstract}
Background. Daodi medicinal material is widely used in Chinese herb medication. However, there is a lack of systematic methodology for identifying characteristics associated with good quality and reliable efficacy of Daodi med-material. Purpose. The purpose of this study is to provide some evidence to further substantiate the use of Daodi medicinal materials. Methods. Seven relevant databases were searched before July 2014. Two evaluators were responsible for screening and categorizing the results. The data was analyzed with Microsoft Excel 2007 and SPSS 21.0 statistical software. Results. Overall, 107 articles were systematically analyzed. Of these studies, 55.1\% (59/107) focused on the methodology to assess Daodi med-material, and 38.3\% (41/107) were interested in med-material ingredients, soil physical and chemical properties, and the geological background system (GBS). Only $6.5 \%$ (7/107) of studies were mainly conducted as clinical trials and animal experiments. Conclusion. Comparisons between Daodi and non-Daodi materials have been studied mainly in terms of the ingredients or composition of medical materials, soil physics and chemistry, and the GBS, and some identifying methodologies have been created to identify Daodi attributes. Until now, there is still no consensus of comparison criteria between Daodi and non-Daodi medicinal material. Only a few studies were conducted through animal experiments and clinical trials to determine Daodi superiority.
\end{abstract}

\section{Introduction}

The term "Daodi" medicinal material has often been used in Chinese ethnopharmacology, and it is usually defined as a material that has been screened after a long period of traditional medical practice, growing in a specific region, and associated with a unique production method. Thus, materials with this label are recognized as having high quality and being clinically effective, and they are a reputable hallmark compared to other medicinal materials that are non-Daodi and so forth [1].
The Chinese word "Daodi" accentuates some distinctive higher quality for the medicinal material that grows in a certain area. The pristine exploration can be retraced to the late Eastern Han Dynasty (25-220 CE) with the advent of the earliest Chinese medicine document Divine Husbandman's Classic of Materia Medica (Shennong Ben Cao Jing). In the Tang Dynasty (618-907 CE), the famous scholar Sun Simiao (the renowned Medicinal Material King) proposed in his classic work Thousand Gold Pieces (Qian jin fang) categorizing the original region for producing medicine in the contemporary administrative province and emphasized a concept 
that medicinal material is essentially embedded in soil. Sun Simiao first used the term "Dao," which was the rudimentary concept that later became known as "Daodi." The "Daodi" concept was initially defined in the Chinese medical classic Essentials of Materia Medica Distinctions (Ben cao pin hui jing yao) in the age of the Ming Dynasty (1368-1644 CE) [2]. The medicinal selection methods had in fact been gradually formulated during medical practice for thousands of years and were established as a unique way to identify materials. Being supported by profound Chinese medical theory, this method is still significant in modern times. Furthermore, the Daodi materials are the most thoroughly investigated materials, and they represent a large amount of the market with tremendous economic value. It is reported that there are 200 Daodi materials out of the 500 traditional medicinal materials, yet Daodi materials contribute approximately $80 \%$ to the overall usage [3].

Chinese herbal medicine has been used in China for over 2000 years [4]. In traditional Chinese medicine (TCM), Daodi materials are considered to have high medicinal efficacy [5]. Most of the studies comparing Daodi and non-Daodi materials were from China. The theory and practice have been developed over thousands of years. Considering territory distribution, Daodi medicinal material can be categorized into Chuan- (Sichuan) Guang- (Guangdong/Guangxi) Yun- (Yunnan) Gui (Guizhou), Nan (Southern China) Bei(Northern China) Zhe (Zhejiang) Huai (Henan), and Shan(Shanxi) Gan- (Gansu) Qing-(Qinghai) Ning (Ningxia). Each medicinal material is rooted in locations with optimal breeding conditions. Because it is the delicate complexity in a natural land that endows a Daodi material with its perplexing mechanism, it has been conventionalized, probably for an expedient solution, through the use of empiricism to differentiate Daodi and non-Daodi materials. As a result, although Daodi medicinal materials are highly valued and renowned nationwide, until now, there has been a lack of sufficient evidence to corroborate traditional practices during Daodi material selection and to identify the superiority, in terms of either quality or clinical efficacy [6].

The purpose of this study is to provide some evidence to further substantiate the perspective on Daodi medicinal materials. We searched the relevant modern literature to analyze and assess comparative studies of Daodi and nonDaodi materials.

\section{Methods}

2.1. Inclusion/Exclusion Criteria. Studies that compared Daodi Chinese traditional medicinal material and non-Daodi traditional medicine were retrieved and included. Review articles were excluded. Article abstracts that could not be traced to their source data and full-text were also ineligible.

2.2. Search and Retrieval Strategy. Seven databases were searched, including PubMed, EMBASE, Web of Science, the Chinese Biomedical Literature Database (CBM), the Chinese Journal Full-Text Database (CJFD), the Chinese Scientific Journal Full-Text Database (CSJD), and the Wanfang database. The key words included "famous-region drug", "authentic medicinal Medicine", "genuine medicinal material”, "genuine crude drugs", “Daodi” (道地), and “Didao" (地道).

2.2.1. Screening Procedure. Two evaluators (Xin Tian and Yannan Zhou) screened the articles independently by reviewing the title and the abstract. If they reached an agreement that the article met the literature identification standard, the full-text version was sought. Any disagreement was settled by a third evaluator, Yali Liu.

2.2.2. Data Retrieval and Analysis. The data of general characteristics and the overall study/processing report were collected. Two evaluators screened data from each article independently, and any discordance from the search results was discussed or another evaluator consulted to reach a resolution (Yali Liu).

A retrieval form was designed according to the study strategy, which mainly included (1) basic information, such as publication journal and time, academic institution, and study funding and (2) data to compare the Daodi and nonDaodi medicinal materials for possible differences: (1) clinical trials and animal experiments that included basic medicinal material information, methodology, results, and conclusions; (2) medicinal material ingredients, the geological background system (GBS), and different chemical and physical soil properties; and (3) a study methodology for identifying a Daodi material, including general med-material information, methodological contrast, and other aspects. The producing regions of the Daodi and non-Daodi described in the basic information and the sample size in either a clinical trial or an animal experiment were recorded as numbers. The following information was described in the form for data collection: growth mode and material sourcing in the basic information section; the reported dose formation and the dosing methods, as well as ingredients, during clinical trials and animal experiments; and other information needed for morphological comparisons between the materials, for the GBS and for a description of the physical and chemical properties and their final comparison conclusion. The other items or contents were recorded as 1 (described in the article) or 0 (not described in the article).

The data were summarized and analyzed by Microsoft Excel 2007 and SPSS 21.0 statistical software, and all the results were described statistically (frequency and percentage).

\section{Results}

3.1. Search and Retrieval Outcomes. Overall, 849 articles were originally acquired, including 324 written in Chinese and 551 articles in English. After deleting repetitive or redundant information, screening was implemented after perusing the abstract section, 120 articles were consequently confirmed through the literature identification criteria, and the 120 fulltext articles were then evaluated. After scrutiny of the full text, another 13 articles were excluded from the study. The 


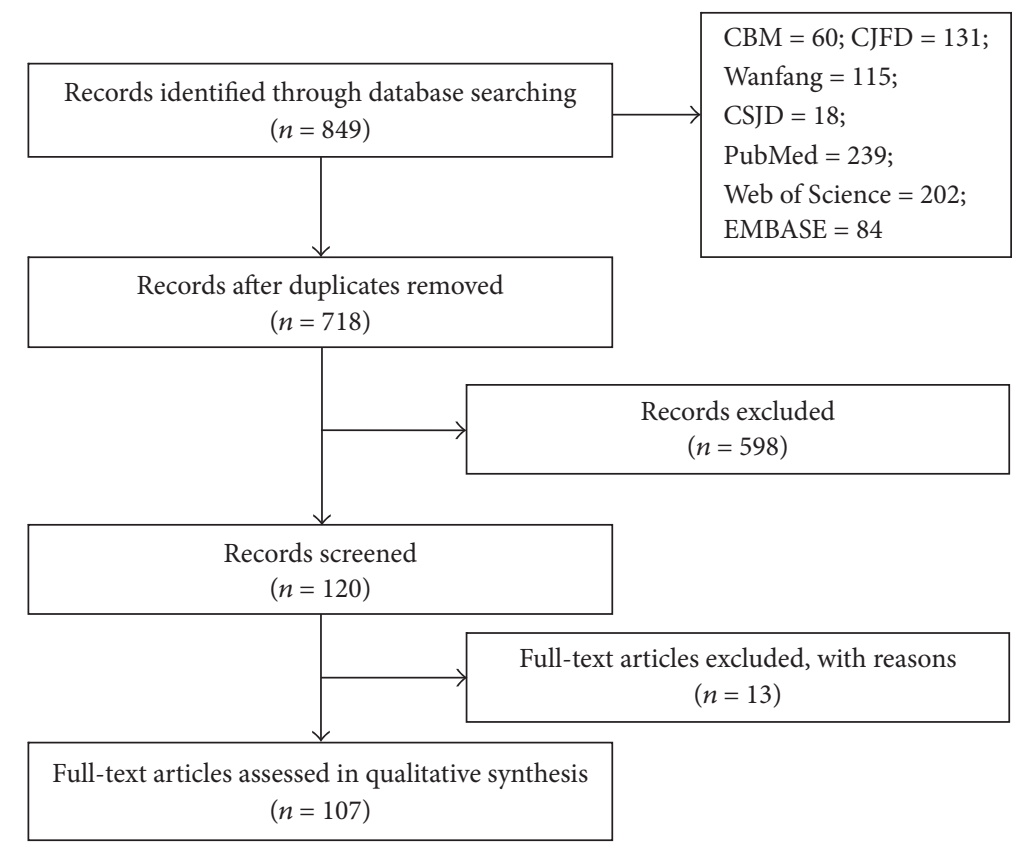

Figure 1: Flow chart of study inclusion.

remaining 107 articles were included, including 4 studies in English (Figure 1).

\subsection{Basic Characteristics of the Included Articles}

3.2.1. Basic Information of Included Studies. This study included 67 (67/107) journal theses, 32 (32/107) academic degree dissertations (30 Master's degree dissertations and 2 Ph.D. dissertations), 5 (5/107) conference papers, and 3 published conference recordings. Ten (10/107) authors, constituting the maximal proportion, were scholars from the China Academy of Chinese Medical Sciences. Of the studies, $58.9 \%(63 / 107)$ had a funding source, the other $41.1 \%(44 / 107)$ had no funding support, and $72.7 \%$ (32/44) were academic degree dissertations. The four English studies reported that there were no conflicts of interest, and not any claims of interest conflict were written in the Chinese articles. Of the 67 journals, the Chinese Journal of Chinese Materia Medica was the most proportional one at 23.9\% (16/67) (Table 1).

The first publication about a Daodi medicinal materials and non-Daodi comparison study was issued as Zhong Yao Cai in 1990. However, from 1990 to 1999, only 3 studies were published, including two studies about traceable mineral elements; the other was a clinical trial. After 2000, there was a continuous increase in the number of relevant studies. The first genetic comparison of the two types of material was published in 2001, and also in this year an animal experiment to investigate the difference in the two types of material was performed. Studies pertaining to the physical and chemical properties of the soil began in 2002. A comparison of medmaterial ingredients, soil physical and chemical properties, and the GBS was initiated.
3.2.2. Study Categorization. Six different categories were summarized in Table 2, including 1 clinical trial, 6 animal experiments, 2 articles pertinent to the GBS and physical or chemical properties of the soil, 24 articles that exclusively investigated medicinal ingredients, 15 articles involving the medicinal composition, the GBS, and the chemical or physical properties of the soil, and 59 articles seeking a methodology to assess non-Daodi materials.

A total of 24 med-materials were included in this investigation and published in the 107 articles. The investigated med-materials were listed below: Pheretima Aspergillum (the only material with a zoological origin), Morindae Officinalis Radix, Atractylodis Rhizoma, Citri Reticulatae Pericarpium, Carthami Flos, Chuanxiong Rhizoma, Rhei Radix et Rhizoma, Salviae Miltiorrhizae Radix et Rhizoma, Moutan Cortex, Angelicae Sinensis Radix, Codonopsis Radix, Rehmanniae Radix, Poria, Aconiti Lateralis Radix Praeparata, Polygoni Multiflori Radix, Magnoliae Officinalis Cortex, Coptidis Rhizoma, Scutellariae Radix, Astragali Radix, Lonicerae Japonicae Flos, Ophiopogonis Radix, Cyathulae Radix, Ginseng Radix et Rhizoma, Notoginseng Radix et Rhizoma, Dioscoreae Rhizoma, Paeoniae Radix Alba, Himalaica Mirabilis, Asari Radix et Rhizoma, Scrophulariae Radix, Polygalae Radix, Alismatis Rhizoma, Anemarrhenae Rhizoma, Aurantii Fructus, and one from a mineral (Gypsum Fibrosum).

\subsection{Comparison of Daodi Medicinal Materials}

3.3.1. General Information of the Origins and Identification of Daodi Medicinal Materials. Because the production regions were divided according to rather inconsistent definitions in these studies, we described producing regions as more than one and only one. More than half of the studies did 
TABLE 1: Basic information of the included studies.

\begin{tabular}{|c|c|c|}
\hline Category & Characteristic & Number (\%) of studies, $n=107$ \\
\hline \multirow{3}{*}{ Year } & 1990-1999 & $3(2.8 \%)$ \\
\hline & $2000-2009$ & $56(52.3 \%)$ \\
\hline & $2010-2014.7$ & $48(44.9 \%)$ \\
\hline \multirow{4}{*}{ Ref type } & Journal thesis & $67(62.6 \%)$ \\
\hline & Academic degree dissertation ${ }^{*}$ & $32(29.9 \%)$ \\
\hline & Conference paper & $5(4.7 \%)$ \\
\hline & Conference recording & $3(2.8 \%)$ \\
\hline \multirow{3}{*}{ Title } & Comparison of Daodi and non-Daodi Medicinal Materials & $21(19.6 \%)$ \\
\hline & Comparison of Different Medicinal Materials & $11(10.3 \%)$ \\
\hline & Others & $75(70.1 \%)$ \\
\hline \multirow{10}{*}{ Author address } & China Academy of Chinese Medical Sciences & $10(9.3 \%)$ \\
\hline & China Pharmaceutical University & $8(7.5 \%)$ \\
\hline & Henan University of Chinese Medicine & $8(7.5 \%)$ \\
\hline & Beijing University of Chinese Medicine & $7(6.5 \%)$ \\
\hline & Chengdu University of TCM & $7(6.5 \%)$ \\
\hline & Hubei University of Traditional Chinese Medicine & $6(5.6 \%)$ \\
\hline & Peking University & $5(4.7 \%)$ \\
\hline & Chinese Academy of Medical Sciences & $4(3.7 \%)$ \\
\hline & Tsinghua University & $4(3.7 \%)$ \\
\hline & Others & $48(44.9 \%)$ \\
\hline \multirow{5}{*}{ Funding source } & Natural Science Foundation of China & $20(18.7 \%)$ \\
\hline & National Basic Research Program of China & $7(6.5 \%)$ \\
\hline & National Administration of Traditional Chinese Medicine Fund Projects & $5(4.7 \%)$ \\
\hline & Support fund not indicated & $44(41.1 \%)$ \\
\hline & Others & $31(29.0 \%)$ \\
\hline Competing interests & Not mentioned & $103(96.3 \%)$ \\
\hline \multirow{5}{*}{ Journal $(n=67)$} & China Journal of Chinese Materia Medica & $16(23.9 \%)$ \\
\hline & Chinese Pharmaceutical Journal & $4(6.0 \%)$ \\
\hline & Journal of Chinese Medicinal Materials & $4(6.0 \%)$ \\
\hline & Lishizhen Medicine and Materia Medica Research & $3(4.5 \%)$ \\
\hline & Others & $40(59.7 \%)$ \\
\hline
\end{tabular}

*Some authors were affiliated with different research institutions, and the first affiliated institution was used; academic degree dissertations were categorized according to the university or college.

not describe the growth mode. The majority of the studies $(78.5 \%, 84 / 107)$ provided information about the process of acquiring med-materials. For example, procurement from a certain Chinese traditional medicine market or going to the original region for purchase was described by some professors or labs. The med-materials were identified by some experts in only $35.5 \%$ (38/107) of the articles. No study specified identification methods in detail. Some studies only mentioned that the med-material was identified; however, there was often no record of any professional assigner, and in some cases, the material extraction process was erroneously considered as the identification process. Of the studies, $52.3 \%$ (56/107) described specific timing about natural medmaterial reaping (at least roughly for a specified lunarmonth). Most of the studies described the basic properties, pharmacological activity, and/or documented efficacy of the Chinese medicine (Table 3).
3.3.2. Data from Clinical Trials and Animal Experiments. Only 6 studies were designed with animal experiments to compare Daodi and non-Daodi materials. One clinical trial investigated differences in the effectiveness between the two material types. The first clinical study was published in 1990, and it compared the effectiveness and safety between rhubarbs. Daodi or non-Daodi materials were used to treat upper gastrointestinal hemorrhage, and the results demonstrated that the Daodi rhubarb had a more effective cure rate and reduced adverse events. Most of the animal experiments in both mice and rats (83.3\%) were published after 2010, with $16.7 \%(1 / 6)$ describing in vitro experiments and $83.3 \%$ (5/6) describing in vivo experiments. The sample size varied from 42 to 140 (mean 96). All the in vivo experiments included randomization into groups, and an aqueous extract was administered most often. All these studies reported pharmacodynamics results, and there were no adverse events. 
TABLE 2: Study content categorization of the included studies.

\begin{tabular}{lcc}
\hline Category & Characteristic & Number (\%) of studies, $n=107$ \\
\hline & Clinical trial & $1(0.9 \%)$ \\
Animal experiment & $6(5.6 \%)$ & $2(1.9 \%)$ \\
Contents of article & GBS and soil physical/chemical properties & $24(22.4 \%)$ \\
& Medicinal composition & $15(14.0 \%)$ \\
& Medicinal composition, GBS, and soil physical-chemical property & $59(55.1 \%)$ \\
\hline Methodology to ascertain Daodi & $13(12.1 \%)$ \\
Atractylodis Rhizoma, Lonicerae Japonicae Flos & $9(8.4 \%)$ \\
Angelicae Sinensis Radix & $8(7.5 \%)$ & $7(6.5 \%)$ \\
Dedicinal species & Dioscoreae Rhizoma & $6(5.6 \%)$ \\
Scutellariae Radix, Achyranthis Bidentatae Radix & $5(4.7 \%)$ & $4(3.7 \%)$ \\
Salviae Miltiorrhizae Radix et Rhizoma & $3(2.8 \%)$
\end{tabular}

TABLE 3: General information on Chinese traditional medicinal materials.

\begin{tabular}{|c|c|c|}
\hline Category & Characteristic & Number (\%) of studies, $n=107$ \\
\hline \multirow{3}{*}{ Number of the Daodi medicinal material original areas } & 1 & $44(41.1 \%)$ \\
\hline & $>1$ & $56(52.3 \%)$ \\
\hline & Not mentioned & $7(6.5 \%)$ \\
\hline \multirow{3}{*}{ Number of the non-Daodi medicinal material origin areas } & 1 & $10(9.3 \%)$ \\
\hline & $>1$ & $90(84.1 \%)$ \\
\hline & Not mentioned & $7(6.5 \%)$ \\
\hline \multirow{3}{*}{ Growth mode of the Daodi medicinal material } & Artificial feeding & $41(38.3 \%)$ \\
\hline & Feral & $19(17.8 \%)$ \\
\hline & Not mentioned & $55(51.4 \%)$ \\
\hline \multirow{3}{*}{ Growth mode of the non-Daodi medicinal material $^{\#}$} & Artificial feeding & $49(45.8 \%)$ \\
\hline & Feral & $19(17.8 \%)$ \\
\hline & Not mentioned & $58(54.2 \%)$ \\
\hline \multirow{2}{*}{ Medicine acquirement } & With origin, without source & $26(24.3 \%)$ \\
\hline & With origin and source & $84(78.5 \%)$ \\
\hline \multirow{2}{*}{ Whether it has been identified } & Yes & $38(35.5 \%)$ \\
\hline & No & $69(64.5 \%)$ \\
\hline \multirow{2}{*}{ Specific time for reaping } & Described & $56(52.3 \%)$ \\
\hline & Not described & $51(47.7 \%)$ \\
\hline \multirow{2}{*}{ Basic property } & Described & $91(85.0 \%)$ \\
\hline & Not described & $16(15.0 \%)$ \\
\hline \multirow{2}{*}{ Pharmacological activity and documented efficacy record } & Described & $89(83.2 \%)$ \\
\hline & Not described & $18(16.8 \%)$ \\
\hline
\end{tabular}

One study included two med-materials; either the Daodi or the non-Daodi was produced in more than one region, which resulted in $>100 \%$. ${ }^{*}$ Of the basic information, some of the med-materials had been recorded with more than one item about growth mode and material origin, which also produced greater than $100 \%$.

The majority of these studies $(83.3 \%)$ reported that the Daodi materials were more effective than the non-Daodi materials, and $25 \%$ reported that the two med-materials had similar effective results.
3.3.3. Medicinal Material Ingredients, Soil Properties, and the GBS. Forty-one studies investigated the med-material ingredient, soil properties, and GBS. Two studies compared the GB and soil properties, 24 studies were exclusively 
about the material ingredients, and 15 articles included all the subjects mentioned above. Three articles examined the different arable soils (different region) that were selected to raise the same herbs, and the possible effect of changes in some inorganic or mineral elements could be analyzed. In this review, we put this article in the category of covering all subjects. For expediency, we added associated study subjects, which included endophyte microbes and metabolites, morphological contrast, and germplasm resource and genetic analysis, Table 4.

Med-material ingredient comparisons were performed mostly to examine mineral and organic compositions. Of these, $63.4 \%(26 / 41)$ compared the active ingredients of an organic composition, and one study investigated pesticide residue, heavy metal substances, and the ineffective ingredients (phenylformic acid) of two med-materials. Overall, $58.5 \%$ (24/41) analyzed mineral element discrepancies, and $4.9 \%(2 / 41)$ reported the probable influence on pharmacodynamics from these minerals. For organic ingredient comparison, many of the studies were focused on the active component of med-materials. The difference of volatile oil between Atractylodis Rhizoma and Chuanxiong Rhizoma was the most frequently analyzed, accounting for approximately $38.5 \%$ of studies (10/26). Some studies examined extracts, polysaccharides, and total ash proportions as the main discrepant indicators between the Daodi and nonDaodi materials. Morphological contrasts were presented in $26.8 \%$ (11/41) of the studies, including physiological anatomy or functional observation. Two studies were conducted to contrast the microanatomy, and one observed some ultramicrostructures.

Some scholars observed the constituents, soil features, and GBS to try to find possible differences in certain medmaterials. Mineral quantities were analyzed in $36.6 \%(15 / 41)$ of the studies, and $22.0 \%$ (9/41) examined the $\mathrm{pH}$ of soil samples. Most GBS studies were conducted to determine geographic characteristics of growing locations, such as altitude and latitude. There were also considerable studies about climatological parameters (i.e., type of climate or conditions, annual sunshine duration, rainfall, and temperature) and the geological background (topography and landforms). A few studies $(2.4 \%, 1 / 41)$ addressed concerns about wind velocity and the humidity of different breeding places (Table 4 ).

3.3.4. Methods for Identifying Daodi Med-Material. Most of the methods used to define a Daodi med-material have depended on physics, chemistry, and biological techniques, as well as statistics, to distinguish apparently similar natural materials, or different production, processing, or concoction procedures have been analyzed to further investigate effective components that might have been affected by processing (Table 5). Fifty-five studies described a methodology to identify a Daodi material, and most of these involved a fingerprint technique. These techniques included chromatography, spectrometry, MRI (magnetic resonance imaging), and DNA fingerprint. Thin-layer chromatography (TLC), highperformance liquid chromatography (HPLC), and gas chromatography (GC) were used. Diode array detector (DAD),
Fourier Transform Infrared (FT-IR), and Near Infrared (NIR) were used for spectrometry. MS was the only MRI analysis. HPLC has been the most frequently used approach, with $50.8 \%$ (30/59), and the other widely used techniques included GC-MS and HPLC-DAD. Fingerprint DNA was reported in 11 studies. The profile of fingerprint DNA in Daodi medmaterials was established in $37.3 \%$ (22/59) of the included studies. Cluster analysis was the most frequent method for statistical analysis $(52.5 \%, 31 / 59)$. Another $8.5 \%(5 / 59)$ included principal component analysis (PCA) processing. Five (8.5\%) studies investigated a process to prepare, produce, and concoct a medicine, which might also contribute to identifying the quality of Daodi.

3.3.5. Results and Clinical Practices. Several studies were investigated in this review. Of these studies, 64.5\% (69/107) claimed that Daodi med-materials were superior to nonDaodi med-materials. Only a small number, $1.9 \%(2 / 107)$, suggested that statistical significance could not be found. Additionally, 2.8\% (3/107) of these studies analyzed the active ingredients of the materials and discovered that the Daodi med-materials had fewer active ingredients. Another 30.8\% $(33 / 107)$ did not make any comparison of superiority or inferiority between the two types of material (Table 6).

\section{Discussion}

4.1. Complicated Environments of Daodi Breeding and Incomplete Studies according to the Identification of a Daodi Med-Material. Previous studies about Daodi med-materials mostly involved medical material tests [7-9], the foundation for formation and development [7, 10-12], quality evaluation and identification/verdicts [13-16], and GAP development [17-22]. In addition, these approaches had mainly been conducted through review and experiment studies. The first study about comparisons was published approximately 20 years ago, and pertinent studies have been increasing gradually. To the best of the author's knowledge, this study is the first review that presents a comparison between Daodi and non-Daodi med-materials.

The pertinent studies on the comparison of Daodi and non-Daodi materials were first published in the 1990s. Beginning in 2000, well-rounded development began again, except for contrasting the ingredients or compositions and pharmacodynamics; other aspects, such as soil characteristics, the GBS, the evaluation methods, and animal experiments, were also inclusively conducted. In 2001, the first exploratory study for some Daodi and non-Daodi genetic comparisons produced a study that has substantiated our molecular insight into these plants. From 2000 to 2010, the published studies were mostly performed through animal experiments, soil analysis, and GBS comparison. Since 2010, the study trends included animal experiments and the introduction of contemporary techniques to identify Daodi and non-Daodi Chinese traditional medicinal material.

When studying med-material ingredients and composition or soil properties, most often, scholars will compare either mineral or organic ingredients. For example, mineral 
TABLE 4: Ingredients of the studied medicine/soil physical and chemical properties/GBS.

\begin{tabular}{|c|c|}
\hline Comparison of medicinal composition, GBS, and soil properties & Number (\%) of studies, $n=41$ \\
\hline \multicolumn{2}{|l|}{ Medicinal composition } \\
\hline \multicolumn{2}{|l|}{ Inorganic elements } \\
\hline Element differences & $24(58.5 \%)$ \\
\hline Detection method & $22(53.7 \%)$ \\
\hline Correlation analysis & $12(29.3 \%)$ \\
\hline Accumulation ability & $11(26.8 \%)$ \\
\hline Character index & $7(17.1 \%)$ \\
\hline Others & $7(17.1 \%)$ \\
\hline \multicolumn{2}{|l|}{ Organic component } \\
\hline Active substance & $26(63.4 \%)$ \\
\hline Extracts & $5(12.2 \%)$ \\
\hline Total ash proportion & $4(9.8 \%)$ \\
\hline Polysaccharide & $4(9.8 \%)$ \\
\hline Others & $6(14.6 \%)$ \\
\hline \multicolumn{2}{|l|}{ Morphologic } \\
\hline Physiological anatomy or functional observation & $11(26.8 \%)$ \\
\hline Microanatomy & $2(4.9 \%)$ \\
\hline Ultrastructure & $1(2.4 \%)$ \\
\hline Germplasm resource & $7(17.1 \%)$ \\
\hline Genetic contrast & $4(9.8 \%)$ \\
\hline Endophyte microbes and metabolites & $3(7.3 \%)$ \\
\hline Others & $6(14.6 \%)$ \\
\hline \multicolumn{2}{|l|}{ GBS and soil physical-chemical properties } \\
\hline \multicolumn{2}{|l|}{ Soil properties } \\
\hline Inorganic elements & $15(36.6 \%)$ \\
\hline $\mathrm{pH}$ & $9(22.0 \%)$ \\
\hline Soil characteristics and type & $8(19.5 \%)$ \\
\hline Available nutrients & $7(17.1 \%)$ \\
\hline Organic material & $6(14.6 \%)$ \\
\hline Physical clay & $4(9.8 \%)$ \\
\hline Soil structure & $3(7.3 \%)$ \\
\hline BS & $3(7.3 \%)$ \\
\hline CEC & $3(7.3 \%)$ \\
\hline Total nutrients & $2(4.9 \%)$ \\
\hline Soil color & $2(4.9 \%)$ \\
\hline Soil moisture & $2(4.9 \%)$ \\
\hline Others & $2(4.9 \%)$ \\
\hline \multicolumn{2}{|l|}{ GBS } \\
\hline Altitude & $8(19.5 \%)$ \\
\hline Latitude & $7(17.1 \%)$ \\
\hline Type of climate or conditions & $7(17.1 \%)$ \\
\hline Annual sunshine duration, rainfall, and temperature & $7(17.1 \%)$ \\
\hline Soil parent material & $5(12.2 \%)$ \\
\hline Topography & $4(9.8 \%)$ \\
\hline Climatic regionalization & $4(9.8 \%)$ \\
\hline Landforms & $3(7.3 \%)$ \\
\hline Vegetation regionalization & $3(7.3 \%)$ \\
\hline Hydrological regionalization & $3(7.3 \%)$ \\
\hline Clay mineral composition & $2(4.9 \%)$ \\
\hline Others & $3(7.3 \%)$ \\
\hline
\end{tabular}


TABLE 5: Methods for assessing Daodi medicinal materials.

\begin{tabular}{|c|c|}
\hline Methods for assessing Daodi medicinal materials & Number (\%) of studies, $n=59$ \\
\hline \multicolumn{2}{|l|}{ Fingerprint } \\
\hline \multicolumn{2}{|l|}{ Chromatography } \\
\hline Thin-layer chromatography/TLC & $4(6.8 \%)$ \\
\hline High-performance liquid chromatography (HPLC) & $30(50.8 \%)$ \\
\hline Gas chromatography (GC) & $10(16.9 \%)$ \\
\hline \multicolumn{2}{|l|}{ Spectrometry } \\
\hline Diode array detector (DAD) & $8(13.6 \%)$ \\
\hline Fourier Transform Infrared (FT-IR) & $7(11.9 \%)$ \\
\hline Near Infrared (NIR) & $4(6.8 \%)$ \\
\hline \multicolumn{2}{|l|}{ MRI (magnetic resonance imaging) } \\
\hline Mass spectra (MS) & $12(20.3 \%)$ \\
\hline \multicolumn{2}{|l|}{ DNA fingerprint } \\
\hline Randomly amplified polymorphic DNA (RAPD) & $5(8.5 \%)$ \\
\hline Amplified fragment length polymorphism (AFLP) & $1(1.7 \%)$ \\
\hline Intersimple sequence repeat (ISSR) & $1(1.7 \%)$ \\
\hline Expressed sequence tags-simple sequence repeat (EST-SSR) & $1(1.7 \%)$ \\
\hline Polymerase chain reaction (PCR) & $5(8.5 \%)$ \\
\hline Characteristic fingerprint & $21(35.6 \%)$ \\
\hline \multicolumn{2}{|l|}{ Statistical analysis } \\
\hline Cluster analysis & $31(52.5 \%)$ \\
\hline Principal component analysis (PCA) & $5(8.5 \%)$ \\
\hline Processing methods & $5(8.5 \%)$ \\
\hline Others & $7(11.9 \%)$ \\
\hline
\end{tabular}

TABLE 6: Results and clinical practice.

\begin{tabular}{lc}
\hline Characteristic & Number (\%) of studies, $n=107$ \\
\hline Results & $69(64.5 \%)$ \\
Daodi medicinal materials were better than non-Daodi medicinal materials & $2(1.9 \%)$ \\
No difference between Daodi medicinal material and non-Daodi medicinal material & $3(2.8 \%)$ \\
Non-Daodi medicinal materials were better than Daodi medicinal materials & $33(30.8 \%)$ \\
No clear comparison & $98(91.6 \%)$ \\
Use in clinical practice & $9(8.4 \%)$ \\
Having a significant use for clinical practice after finding that the genuine material is distinguishable & \\
from the nongenuine material and confirming the quality for the GAP (good agricultural practice) system & \\
$\quad$ Not mentioned &
\end{tabular}

differences in the two categories of med-materials were usually investigated. However, only a few of these studies established certain mineral characteristic as a significant indicator for claiming authenticity.

The GB is defined as the specific synthesis of attributes in a geologic body and geologic agents that are highly related to the med-materials, including quaternary sediment, the mineral distribution or rock mass, tectonics, crustal movement, geographic and geomorphic factors, topography and landforms, geochemistry, hydrogeology, and other multifarious considerations. In fact, the GBS is defined as a GB 2-dimensional integrality (GB, climate, biology, etc.), which is part of modern system theory about the foundation of natural nonequilibrium open system consistence law [9]. Most of the studies in this review are about the original production regions in terms of their geographic features, climate data, GB, and so on. Regarding differences in pivotal ingredients, the pharmacodynamics of the two types of medmaterial have high relationships with the geological milieu. Considering the complications in geographic environments, there are remarkable discrepancies in various soil and water conditions, climates, sunlight durations, and distributions of biological beings. All these factors may affect the quality of the med-materials, and as they may play a role in the differences in medical efficacy, these issues require further clinical trials to provide substantial evidence of these effects.

A majority of the studies to identify Daodi Chinese traditional medicinal material used a fingerprint method, and HPLC was the second most common approach. Many 
studies identified the med-materials via only one method for fingerprint examination, and a combination of two methods for fingerprint study was also frequently applied, especially GC-MS and HPLC-DAD [19]. Few studies were facilitated by DNA fingerprint graphing. However, there are great variations in applying specific methods. In addition, NMR was also used in the analysis of plant extracts [20].

\subsection{The Current Challenges, Issues, and Prospective Solutions.} Even among the wide range of data sources sought in this review study to compare the overall investigational results between Daodi and non-Daodi materials, only 34 medmaterials were covered, which contrasts with the more than 200 Daodi materials that have been recorded. This outcome suggests that other materials have not been sufficiently investigated. Ingredient differences were unanimously considered to be one of the standards for claiming superiority. However, for many studies in this review, a view of the growth mode was not taken for either Daodi or non-Daodi med-materials; further investigation on specific provenances, sourcing status, and reaping time was not considered; there was no report about whether an experimental identification was conducted between the two types of material; and there was a similar deficiency for articulating the processes and methods to claim a difference. In fact, this information has great significance in regard to comparing the functional ingredients of Daodi and non-Daodi materials as well as clinical effectiveness. Therefore, it is highly recommended that producing region information be considered for clarification. In cases regarding the necessary identification of the attributes of some medmaterials, experts and professionals should be invited to consult. If so, the study's accuracy would be enhanced with reliable and intelligible scientific evidence, which would benefit the development of future translational medical studies.

Suitable geographical conditions are exterior factors for producing Daodi ingredients [1]. Admittedly, the Chinese geological environment has evolved on a large scale over time and under natural conditions. As a result, it may play a role in the transference of the Daodi med-material producing region. However, the fundamental principle for trustworthiness of Daodi med-material has always been stated as "Good Quality and High Efficacy" for its label and "Foundation on the Primacy" [23] for its essence. Two studies [24, 25] included in this review have distinguished the original region using the "ancient" tract and "current" tract, and both are attributed to the Daodi category, which disregards a possible effect on the medicine quality when considering the changes in the methods applied for producing region division or categorization.

Until now, clinical discrepancies were derived from empiricism rather than from more substantial trial results. In addition, modern studies still take material ingredients, soil properties, and the GBS as the investigational cynosure. Studies have seldom been performed through animal experiments and clinical practical tests. Furthermore, both of these types of study have only been sparsely and fragmentally reported. In most of the real cases and dosing in Chinese traditional medicine, a combination recipe with different materials was prescribed, and it was seldom administered as just a single medicinal ingredient/dose. Because there are interactions between different medicine ingredients and agents, with different mechanisms that could complicate the results, some scientists consider that even clinical trials, which are a widely accepted study method, cannot be taken as a valuable way to interpret whether any single medicine from different producing areas can be differentiated or confirmed to have an exclusive effectiveness. There might be only some slight or subtle difference between the Daodi and non-Daodi materials that is not as remarkable as the contrast between a medicinal agent and a placebo in established Western clinical pharmacology. As a result, the study has to include many more subjects to present a difference, which therefore poses a great challenge to obtaining clinical testimony. None of these included studies has investigated toxicity and adverse events, and there is evident incompleteness of data for toxic comparison between the medicinal materials. If the effectiveness of Daodi and non-Daodi materials in animal models is to be compared, it has been reasonably suggested that some parallel studies with a well-controlled design, as well as with wholeness and precision, be preferably established to investigate the relevant toxicity to further dig deeply into the discovery of the pharmacodynamics and safety profile.

One certain medicinal material may be produced from different regions, and its active ingredients and quantities can vary. Active ingredients or components are a uniquely important attribute for any Daodi med-material [23]. However, it is necessary to note here that a quality verdict might not be proportionally related to certain chemical compositions. In fact, certain levels of some ingredients cannot substantially indicate a remarkable or significant difference between one Daodi and another non-Daodi material [26]. Endophytes are some fungi or bacteria that have been living in healthy plant tissues or organs throughout the plants' whole life or some special growth stage; in addition, endophytes can beneficially play a role in some formation or accumulation of active ingredients [27]. Derived metabolites are usually regarded as the main ingredients or components in Chinese traditional medicine. The benefits of these derived metabolites have been commonly agreed on, such as disease-resistance, antipest, and counter-environmental adversary effects [28]. Only three studies were conducted on endophytes and compared derived metabolites in Chuanxiong Rhizoma and Lonicerae Japonicae Flos to investigate their Daodi attributes [29-31].

The superior germplasm resource is an internal factor of the formation of a Daodi medicinal material [1], and for this reason, many modern studies compare soil properties, such as mineral differences in producing soil that may breed a Daodi or non-Daodi material. However, there is still a lack of evidence that necessitates some further studies to investigate the relationships between soil minerals and the medicine toxicity, and the compound minerals that may be formed into any quality, as well as med-material yields, especially indispensable minerals that could improve the quality of traditional medicine, are still an open issue. We suggest that these aspects require further study to clarify the association between soil minerals and the chemical composition of the med-materials as well as to determine 
if pragmatic significance was involved in the process of cultivation and quality assurance, along with fertilization for time and quantity considerations [32]. All the comparisons of the soil properties and various environmental factors in this study to ascertain significant differences between Daodi and non-Daodi med-materials are designed to discover natural conditions that formulate and compose Daodi supreme qualities. However, there is a lack of objective and clear methods as well as standards to produce reliable contrasts. Therefore, these pertinent studies have unfortunately been bogged down in a perplexing impasse, "purposed for a study, but without any credible measurement" and "wheeling into repetitive inanity." As a consequence, it is very difficult to disclose any ecological factor to identify a Daodi material. From this review, we recognize that assessment methods and standards must be established if the relationship between Daodi attributes and the ecological environment is to be studied [26].

Morphological comparison between Daodi and nonDaodi med-materials is one of the key aspects; however, it often highlights physiological states directly from observations, appearances, or phenomena, which usually come from individual empirical knowledge and are probably prejudiced by different viewpoints subjected to a provincial judgment. Attributed to these incomplete and nonquantified standards, these methods can be applied and developed with limitations.

Recently, new technologies and methods have been developed rapidly to be used for Daodi med-material identification, and these approaches will hopefully overcome some difficulties of traditional methods for identifying differences. Shilin Chen suggested that DNA barcoding [33] can be used for med-material sample identification when there is little background information. This method is advantageous for methodological generalization and digitalization as well as developmental feasibility [34]. He also proposed the Herb Genome Project (HerbGP) (Chen et al., 2010) and established a DNA barcoding [35] system, which has great potential. Xiaohe Xiao suggested establishing a "synergic Daodi medmaterial standard" (Daodi Indicator, DDI), and this method might be more systematic and objective for a control assessment as well as for confirming high quality med-materials [36] (Xiao et al., 2012). Xueyong Wang proposed some more systematic and precise methods for Chinese traditional medicine study to produce reliable evaluations [37]. In 2002, a complicated systematic theory about the Chinese traditional medicinal material GAP tool was suggested by Luqi Huang [17]. The author applied modern physics, chemistry, biology, and statistical methods to identify ingredients and confirm the quality of two med-materials, even though no agreement was reached.

4.3. Limitations of This Study. Above all, only seven Chinese or English databases were included as data sources, and the final studies included were entirely written in Chinese. No English articles were retrieved. Second, this review is only based on modern studies, and no associated provenance from ancient studies has been retraced. The third limitation is that it is possible to miss some studies because the designated keywords have not been included anywhere in an article's title, abstract, and keyword list, especially in cases where there was some specific description with different vocabularies, although this study began with a systematic retrieval method and plan.

\section{Conclusion}

Comparisons between Daodi and non-Daodi materials have been studied mainly in terms of med-material ingredients or composition, soil properties, the GBS, and some identifying methodologies to assess Daodi attributes. These factors are closely related to med-material production and effective ingredient identification, yet these factors are incapable of providing direct evidence to demonstrate safety and effectiveness. Some studies applied modern biomedicine or biostatistics methods for quantity analysis to compare the two types of med-material, even though there is still no consensus of comparison criteria between Daodi and nonDaodi medicinal material. Until now, only a few studies were conducted through animal experiments and clinical trials to discern the superiority of Daodi. These results highly suggested that clinical trials and fundamental studies are needed to explore the effectiveness and safety profiles as well as to further translate the benefits of clinical Chinese medicine into practice.

\section{Conflicts of Interest}

All authors declare that they have no conflicts of interest.

\section{Authors' Contributions}

Xingyue Yang, Yali Liu, and Liyun He conceived and designed the study. Yali Liu, Xin Tian, and Yannan Zhou searched for data. Xin Tian, Yannan Zhou, Xingyue Yang, Xinlong Li, Tingting $\mathrm{Lu}$, and Changhe Yu extracted the data and assessed the reporting quality of the animal experiments. Xin Tian and Yali Liu analyzed the data. Yali Liu and Liyun He interpreted the data. Xingyue Yang, Xin Tian, and Yali Liu participated in writing the manuscript. All authors read and approved the final manuscript.

\section{Acknowledgments}

This study was supported by the State Key Laboratory Breeding Base of Dao-Di Herbs (no. z0310-3) and Central Level Major Expenditure Increase and Decrease Project (2060302).

\section{References}

[1] Z. Zhao, P. Guo, and E. Brand, "The formation of daodi medicinal materials," Journal of Ethnopharmacology, vol. 140, no. 3, pp. 476-481, 2012.

[2] F. Liang, J. Li, W. Zhang, and R.-X. Zhang, "Brief introduction of geo-authentic herbs," China Journal of Chinese Meteria Medica, vol. 38, no. 9, pp. 1455-1457, 2013. 
[3] S. Y. Qin, X. H. Xiao, L. Y. Li, Z. W. Su, Q. M. Fang, and C. Z. Qiao, "Study on the distribution characteristics of the genuine traditional drugs," Resource Development and Market, vol. 13, no. 6, pp. 261-264, 1997.

[4] A. Flower, G. Lewith, and P. Little, "Combining rigour with relevance: A novel methodology for testing Chinese herbal medicine," Journal of Ethnopharmacology, vol. 134, no. 2, pp. 373-378, 2011.

[5] B. Liu, Z.-Y. Guo, R. Bussmann et al., "Ethnobotanical approaches of traditional medicine studies in Southwest China: A literature review," Journal of Ethnopharmacology, vol. 186, pp. 343-350, 2016.

[6] The Xiangshan Science Conferences, The 390th Xiangshan Science Conferences Session: Systematic Study of Chinese Geoherbs and Its Quality Characteristic and Cause of Formation, 2013, http://www.xssc.ac.cn/ReadBrief.aspx?ItemID=140.

[7] F. Liang, J. Li, W. Zhang, and R.-X. Zhang, "Causes for change in producing areas of geo-authentic herbs," China Journal of Chinese Meteria Medica, vol. 38, no. 10, pp. 1649-1651, 2013.

[8] X. H. Xiao, S. L. Chen, L. Q. Huang, and P. G. Xiao, "Survey of investigations on Daodi Chinese medicinal materials in China since 1980s," China Journal of Chinese Meteria Medica, vol. 34, no. 5, pp. 519-523, 2009.

[9] W. Z. Xie, "Study on Daodi Chinese medicine," Journal of Traditional Chinese Medicine, vol. 10, pp. 43-46, 1990.

[10] L. Q. Huang, L. P. Guo, J. Hu, and A. J. Shao, "Molecular mechanism and genetic basis of geoherbs," China Journal of Chinese Meteria Medica, vol. 33, no. 20, pp. 2303-2308, 2008.

[11] X. Meng, S. Chen, and X. Wang, "Dao-di herbs and its change of cultivated origin place," China Journal of Chinese Meteria Medica, vol. 36, no. 13, pp. 1687-1692, 2011.

[12] C. Peng, "The form essentials and crisis of genuine medical herbs," Pharmacy and Clinics of Chinese Materia Medica, vol. 2, no. 1, p. 10, 2011.

[13] L. P. Guo and J. H. Liu, "The naphtha composing characteristics of geoherbs of atractylodes lancea," China Journal of Chinese Materia Medica, vol. 27, no. 11, pp. 13-18, 2002.

[14] H. L. Huang, K. L. Liu, F. Shao, G. Ren, F. Duan, and R. H. Liu, "Studies on HPLC fingerprint of heishunpian from different origin," Chinese Journal of Experimental Traditional Medical Formulae, vol. 17, no. 16, pp. 44-49, 2011.

[15] Z. X. Xiang and S. L. Gao, "Application and study of HPLC fingerprint of Lonicera japonica for identi6cation of geoauthenticity," China Journal of Chinese Meteria Medica, vol. 33, no. 9, pp. 996-998, 2008.

[16] K. Tong, The Quality Assessment of Cyathula officinalis Kuan, Sichuan Agricultural University, Sichuan, China, 2013.

[17] L. Q. Huang and D. Cui, Study on Complex System of Chinese Materia Medica GAP Fulfilling. China Journal of Chinese Meteria Medica, vol. 27, no. 1, p. 5, 2002.

[18] M. X. Ran and H. Q. Zhou, "Chinese material medical regionalization with Chinese material medica and area economic development," Journal of Chinese Medicinal Materials, vol. 38, no. 4, pp. 655-658, 2015.

[19] H. Sheridan, L. Krenn, R. Jiang et al., "The potential of metabolic fingerprinting as a tool for the modernisation of TCM preparations," Journal of Ethnopharmacology, vol. 140, no. 3, pp. 482-491, 2012.

[20] J.-L. Wolfender, K. Ndjoko, and K. Hostettmann, “The potential of LC-NMR in phytochemical analysis," Phytochemical Analysis, vol. 12, no. 1, pp. 2-22, 2001.
[21] Y. M. Tang and F. Wang, "Geoherbs and good agriculturing practice," Lishizhen Medicine and Materia Medica Research, vol. 15, no. 6, pp. 361-362, 2004.

[22] S. M. Wang, Y. X. Cui, M. X. Wu, and S. W. Liang, "The quality evaluation of the different collection periods and processing technologies of floslonicerae in henan gap," in Lishizhen Medicine And Materia Medica Research, vol. 18, pp. 1061-1063, Lishizhen Medicine And Materia Medica Research, 2007.

[23] X. P. Liu and L. Li, "Relation between authentication of hers and their active constituents," Research and Practice of Chinese Medicines, vol. 18, pp. 24-29, 2004.

[24] Z. W. Wu, Z. N. Wang, Y. Lu, D. Wang, R. Q. Qian, and M. Y. Shang, "Comparativestudy on anti-inflammatory and antipyretic effects between Dao-di herb and non Dao-di herb of Huangqin," China Journal of Chinese Meteria Medica, vol. 37, no. 23, pp. 3628-3632, 2012.

[25] D. Wang, Q.-Y. Zhang, X.-X. Yang et al., "Study on HPLCfingerprint-based identification of Dao-di herb and non Daodi herb of Scutellariae Radix," China Journal of Chinese Meteria Medica, vol. 38, no. 12, pp. 1951-1960, 2013.

[26] D. Yan, J. B. Wang, J. X. Li, L. N. Ma, and X. H. Xiao, "Stratery for research on quality identification and ecological environmentrelated of Dao-di herb," China Journal of Chinese Materia Medica, vol. 37, no. 17, p. 2672, 2012.

[27] M. L. Chen, L. Q. Huang, S. H. Ouyang, A. J. Shao, and S. F. Lin, "Influences of plant endophyte on genuine medicinal materials," Chinese Journal of Information on TCM, vol. 13, no. 9, pp. 40-42, 2006.

[28] L. Q. Huang and L. P. Guo, "Secondarymetabolites accumulating and geoherbs formation under enviromental stress," China Journal of Chinese Materia Medica, vol. 32, no. 4, pp. 277-80, 2007.

[29] H. Wang, The Study on The Effect of The Endophytes to The Quality of Ligusticum Chuanxiong Hort (Umbellales), Chengdu University of TCM, Sichuan, China, 2012.

[30] Y. L. Wang, Corretation study on endoPhyte and quality of Rhizoma Chuanxiong, Chengdu University of TCM, Sichuan, China, 2008.

[31] H. D. Ye, Study on The Correlation between Endophytic Fungus with Metabolites in Lonicera Japonica Thunb, Henan University of Traditional Chinese Medicine, Henan, China, 2012.

[32] Z. R. Gu, F. G. Shi, and Y. Jin, "Advance in study on the relationship between soil inorganic elements and quality of traditional chinese medicine," Guangdong Weiliang Yuansu Kexue, vol. 20, no. 11, pp. 18-22, 2013.

[33] P. D. N. Hebert, A. Cywinska, S. L. Ball, and J. R. DeWaard, "Biological identifications through DNA barcodes," Proceedings Biological sciences/The Royal Society, vol. 270, no. 1512, pp. 313321, 2003.

[34] S. L. Chen, B. L. Guo, G. J. Zhang, Z. Y. Zhang, G. M. Luo, and S. Q. Sun, "Advances of study on new technology and method for identifying traditional Chinese medicinal meterials," China Journal of Chinese Materia Medica, vol. 37, no. 8, pp. 1043-1055, 2012.

[35] S. L. Chen, Y. Z. Sun, J. Xu, H. M. Luo, C. Sun, and L. $\mathrm{He}$, "Strategies of the study on Herb Genome Program," Acta Pharmacetutica Sinica, vol. 45, no. 7, pp. 807-812, 2010.

[36] X. Xiao, J. Wang, D. Yan, and Y. Lv, "Establishment of Dao-Di index and its significance in quality control and rational usage of Chinese medicine," China Journal of Chinese Meteria, vol. 137, no. 11, pp. 1513-1516, 2012. 
[37] X.-Y. Wang, C.-L. Liao, S.-Q. Liu, C.-S. Liu, A.-J. Shao, and L.-Q. Huang, "A accurate identification method for Chinese materia medica-systematic identification of Chinese materia medica," China Journal of Chinese Meteria Medica, vol. 38, no. 9, pp. 14511454, 2013. 


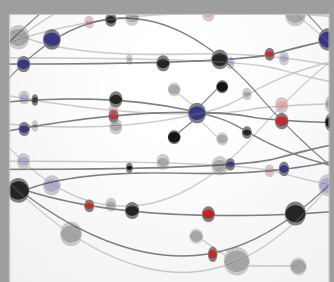

The Scientific World Journal
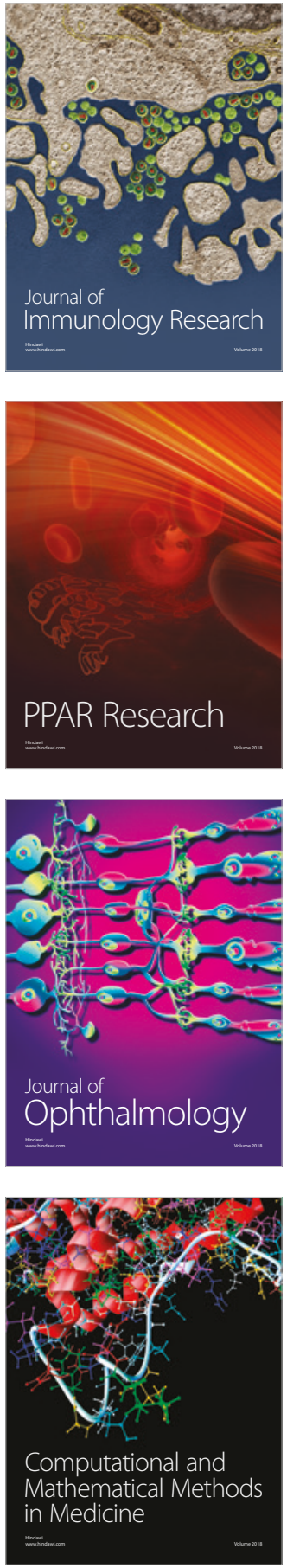

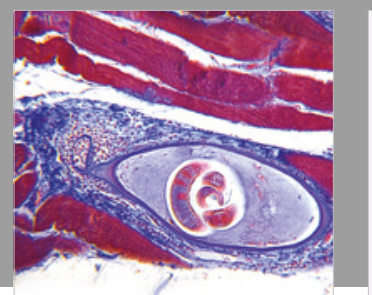

Gastroenterology Research and Practice

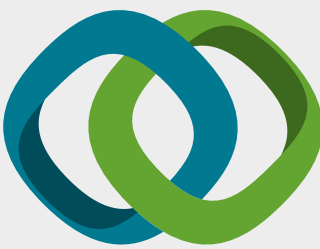

\section{Hindawi}

Submit your manuscripts at

www.hindawi.com
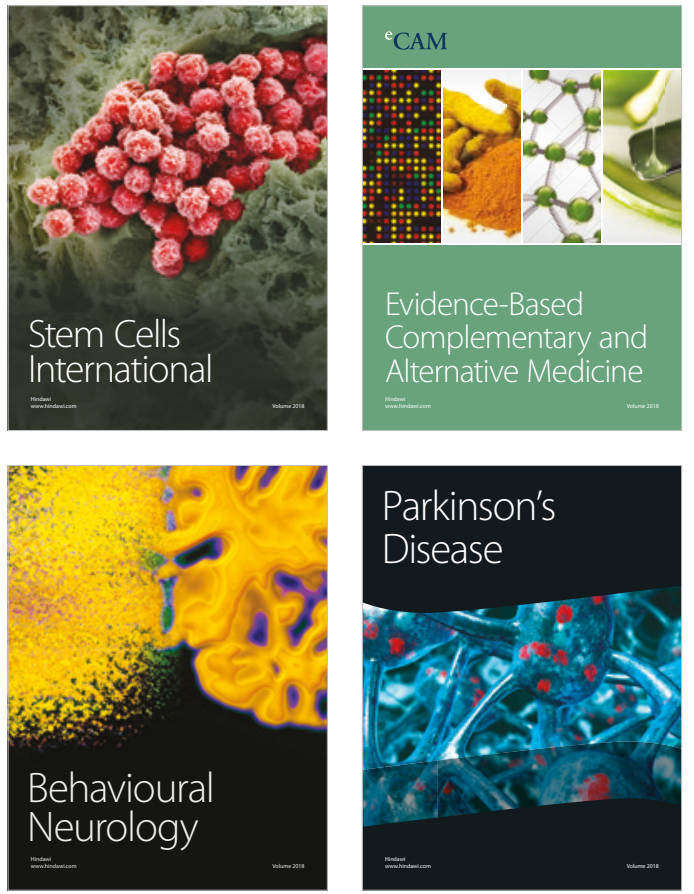

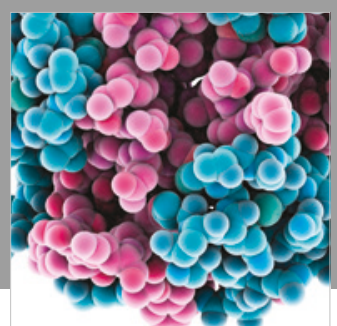

ournal of

Diabetes Research

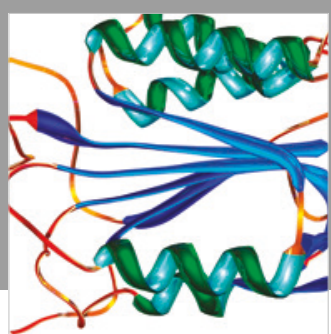

Disease Markers
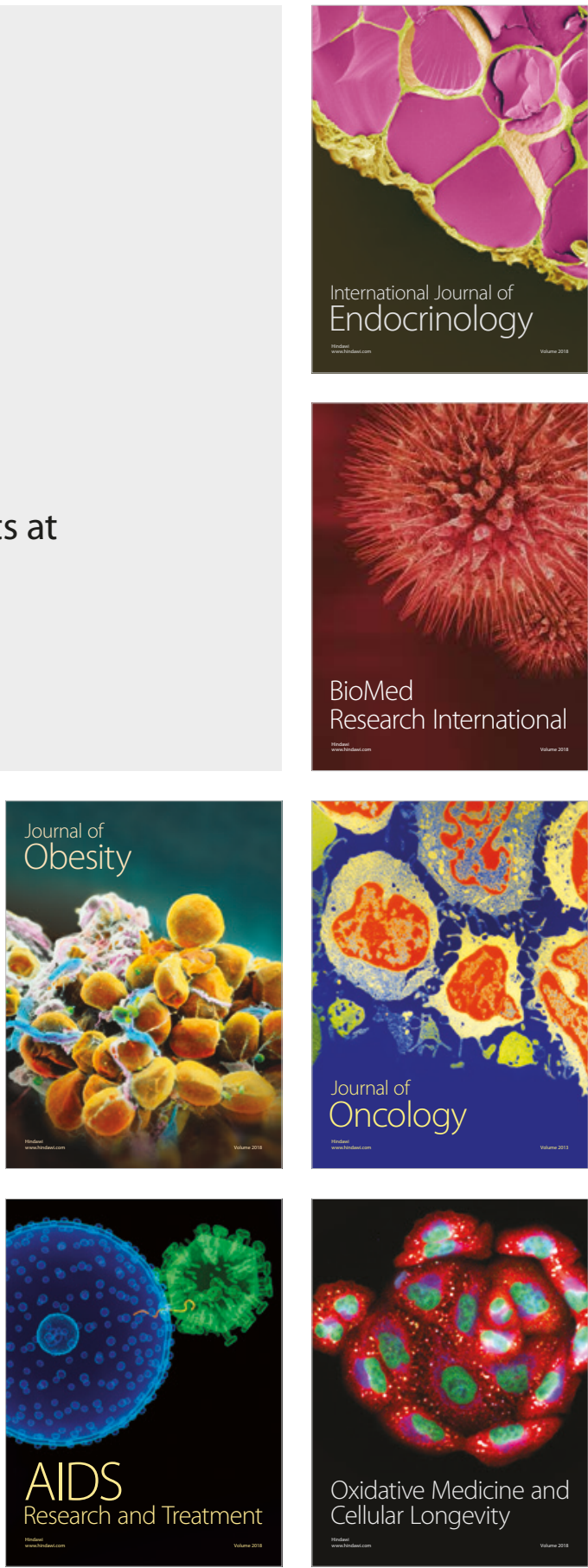